\title{
Relación entre la adicción al ejercicio, el uso de dispositivos fitness y la ansiedad rasgo Relationship between exercise addiction, use of fitness devices and trait anxiety \\ Javier Simón-Grima, Andrés San Martín-Salvador, Nerea Estrada-Marcén, Jaime Casterad-Seral \\ Universidad de Zaragoza (España)
}

\begin{abstract}
Resumen. El reciente auge de la tecnología y su protagonismo en el mundo del fitness han desencadenado la publicación de libros e investigaciones científicas. Además, la reciente preocupación de muchos investigadores por la adicción al ejercicio y sus consecuencias para la salud aumentan la importancia de investigar si estas variables (tecnología, adicción al ejercicio y salud) podrían estar relacionadas de algún modo. Por ello, el objetivo del presente estudio fue investigar si existe o no una asociación entre el uso de dispositivos fitness, la adicción al ejercicio y la ansiedad-rasgo. Para llevar a cabo el presente estudio se analizó una muestra de 102 usuarios de centros deportivos con una media de 30 años de edad, a los cuales se les proporcionó cuestionarios para evaluar el uso de las tecnologías, la adicción al ejercicio (Exercise Addiction Inventory) y el nivel de ansiedad-rasgo (State-Trait Anxiety Inventory). Los resultados mostraron que la mayoría de los sujetos de centros fitness empleaban tecnologías durante sus entrenamientos, siendo mayor el porcentaje de hombres que hacían uso de ellas que de hombres. También se hallaron interesantes correlaciones entre la edad y la adicción al ejercicio físico, así como entre ansiedad-rasgo y adicción al ejercicio físico. Con respecto a qué dispositivos son los más utilizados, los wearables y las apps móviles obtuvieron el mayor porcentaje. No obstante, se hacen necesarias más investigaciones experimentales con muestras más amplias que evalúen un mayor rango de variables asociadas a la salud.
\end{abstract}

Palabras clave: fitness, wearables, adicción al ejercicio, ansiedad, tecnología.

Abstract. Recently, the growth of technology and its prominent importance in the world of fitness have triggered the publication of several books and research papers. Moreover, there are a strong concern about exercise addiction and its health consequences among the research community. Variables such as technology, exercise addiction and health could be related with the issue. This study aims at investigating whether there is an association between the use of fitness devices, exercise addiction and trait anxiety. The investigation analyzes a simple of 102 users of fitness centers located in Zaragoza with a mean age of 30 years old. The study uses different questionnaires in order to evaluate: i) the use of technology (own questionnaire), ii) exercise addiction (Exercise Addiction Inventory) and iii) the level of trait anxiety (State-Trait Anxiety Inventory). Results indicates that most of the users use technology during their training sessions, being larger the percentage of men than women. Further, interesting associations between age and exercise addiction were found, as well as, between trait anxiety and exercise addiction. In addition, the study highlights that wearables and mobile apps are the trendy devices. Nevertheless, greater range of variables associated with health calls for more experimental studies with larger samples.

Keywords: fitness, wearables, exercise addiction, anxiety, technology.

\section{Introducción}

En los últimos años ha habido un importante aumento en el consumo y uso de dispositivos relacionados con la práctica de ejercicio físico. Tanto es así, que algunos expertos apuntan a que el mercado mundial de la tecnología fitness aumentará alrededor de un 30\% hasta el 2021 (Hortas-Rico, Onrubia \& Pacífico, 2014). De acuerdo con estas previsiones, en 2018, el Colegio Americano de Medicina del Deporte (ACSM) anunciaba las tendencias mundiales en el fitness para el 2019, en el que se encontraban las tecnologías portátiles debido al auge de éstas en la actualidad (AladroGonzalvo \& Ibarzábal, 2019). Con respecto a la tecnología más usada por las personas que realizan ejercicio físico, los wearables (smartwatch, pulsómetros, etc.) parecen ser los preferidos por los usuarios debido a su comodidad de uso y de transporte (López-Ruiz, López-Torres, Rodríguez, de Vargas-Sansalvador \& Martinez-Olmos, 2015). También las aplicaciones móviles podrían formar parte de estrategias de intervención para contabilizar la cantidad de ejercicio físico y fomentar la motivación hacia el mismo, debido a que en la actualidad los teléfonos inteligentes tienen un uso generalizado en la sociedad (Smith, 2015). Además, el público objetivo de éste tipo de tecnología es amplio, y no se han observa-

Fecha recepción: 15-03-20. Fecha de aceptación: 07-08-20

Javier Simón-Grima

jsimongrima@hotmail.com do diferencias significativas en cuanto a su utilización según sexo, edad o estatus social (Hughes, Nagendran, Dieker, Hynes \& Welch, 2015).

A pesar de que uno de los objetivos de éste tipo de dispositivos fitness es el de promover hábitos de vida saludables, y de que existe gran cantidad de evidencias científicas en relación a los beneficios que reporta a las personas la práctica de ejercicio físico (Allegre, Therme \& Griffiths, 2007; Kokkinos \& Myers, 2010), muchos investigadores se han centrado en los últimos años en estudiar la adicción al ejercicio, la cual ha sido descrita como un patrón de comportamiento en el cual el individuo no tiene control sobre sus hábitos de ejercicio y actúa de forma compulsiva, presentando una dependencia de la actividad física, lo que acarrea consecuencias a nivel de salud, relaciones personales y profesionales (Szabo, Griffiths, de La Vega Marcos, Mervó \& Demetrovics, 2015). En revisiones sistemáticas y meta-análisis como el realizado por Simón-Grima, Estrada \& Montero (2018) se concluye que el nivel de adicción al ejercicio podría variar en función de la edad y frecuencia de entrenamiento. Además, éstos mismos autores (Op. Cit) también concluyeron que ésta posible adicción al ejercicio podría perjudicar la salud mental y relacionarse a su vez con trastornos de alimentación. Sin embargo, la adicción al ejercicio no ha sido clasificada como un trastorno mental en la edición más reciente (quinta) del Diagnostic and Statistical Manual of Mental Disorders (DSM-5) (American Psychiatric Association, 2013), aunque autores como Egorov y Szabo 
(2013) aseguran que el ejercicio puede ser potencialmente adictivo. Con respecto a las adicciones, Griffiths (1996) creó un modelo en el que se incluían los seis síntomas comunes a cualquiera de ellas (internet, videojuegos, sexo, etc.). Éstos seis componentes son: 1) rasgo sobresaliente, 2) modificación del estado de ánimo, 3) tolerancia, 4) síntomas de abstinencia, 5) conflicto y 6) recaída. En base a éste modelo y basado en los síntomas derivados de la adicción al ejercicio, Szabo y Griffiths (2004) desarrollaron el Exercise Addiction Inventory (EAI) como instrumento de cribado para este trastorno (Griffiths, 1996). El EAI se compone de 6 preguntas basadas en los 6 componentes generales de la adicción (Op. cit).

Esta adicción al ejercicio parece estar relacionada con trastornos alimentarios como la bulimia e incluso la hipocondría (Lejoyeux et al. 2012), la autoestima y las actitudes narcisistas (Bruno et al. 2014), la inestabilidad general que podría llegar a repercutir en la salud (Warner \& Griffiths, 2006) y el aumento de los niveles de ansiedad pre- ejercicio físico (Hollander, 1993). Con respecto a la ansiedad, el concepto de ésta difiere según el autor. No obstante, la gran mayoría de autores coinciden en que se trata de una respuesta de anticipación propia del organismo frente a estímulos que pueden ser externos o internos, que son percibidos por el individuo como una amenazada para su estabilidad, lo cual genera una preocupación excesiva e inapropiada que es persistente y no se limita a circunstancias particulares (Lader, 2015). Para medir la ansiedad hay instrumentos de uso generalizado, como es el caso del State-Trait Anxiety Inventory (STAI), creado por Spielberger, Gorshuch y Lushene en 1968 con el objetivo de evaluar la ansiedad en dos dimensiones: estado y rasgo (Spielberger \& Reheiser, 2009). La ansiedad rasgo, fue conceptualizada por Spielberger como un comportamiento caracterizado por ser estable en el individuo, llevándole a contemplar gran parte de situaciones como una amenaza, de forma que se provoca un aumento notorio de los niveles de ansiedad estado. Por otra parte, la ansiedad estado, de forma contraria a lo que sucede en la ansiedad rasgo, es un estado transitorio de ansiedad, lo que significaría que el nivel de ansiedad va variando según cómo la persona percibe el grado de amenaza que le supone afrontar distintas situaciones.

Se ha observado que la práctica regular de actividad física de forma adecuada puede tener beneficios sobre la salud mental, el estado de ánimo y los síndromes como la depresión y la ansiedad (Martinsen, 2008). Sin embargo, la ansiedad tiene relación en muchas ocasiones a una imagen corporal y autoconcepto negativo, que está relacionado con trastornos dismórficos (Raich, 2004), lo que puede desembocar en una práctica de ejercicio desmedida o excesiva. En relación a ésto, investigaciones como la realizada por Spano (2001) concluyó que la ansiedad rasgo se asociaba con la adicción al ejercicio. Similares resultados se han observado en otros estudios cuando se analizó la ansiedad estado y la dependencia al ejercicio (Li, Nie \& Ren, 2015). No obstante, no se ha observado que, en atletas, ésta ansiedad provoque desórdenes del estado de ánimo (Araújo, De Mello \& Leite, 2007). Además, la mayoría de estudios en el campo de la adicción al ejercicio han investigado la ansiedad estado y pocos la ansiedad rasgo.
De acuerdo con esto, la ansiedad podría estar relacionada o asociada con la adicción al ejercicio. Sin embargo, las evidencias actuales no son consistentes debido a múltiples factores como la diferencia de muestras, metodologías diferentes, etc. (Simón-Grima, Estrada-Marcén \& Montero-Marín, 2018). Por ello, el presente estudio tiene el objetivo de arrojar más luz sobre este tema, buscando una posible asociación en usuarios de centros fitness entre el uso de dispositivos y tecnología, la adicción al ejercicio y la ansiedad rasgo, teniendo en cuenta variables como la frecuencia de entrenamiento, edad, actividad física pasada y género.

\section{Métodos}

\section{Participantes}

El universo de estudio estuvo formado por usuarios de centro fitness Premium de la ciudad de Zaragoza, debido a que ésta es una ciudad idónea para el estudio, ya que el perfil socioeconómico de sus habitantes es representativo a nivel nacional. Además, en Zaragoza ya se han llevado a cabo estudios previos sobre el ámbito del fitness (Estrada-Marcén, Sanz-Gonzalo, Simón-Grima, Casterad-Seral \& Roso-Moliner, 2019; Estrada-Marcén, Sanz-Gonzalo, Casterad-Seral, SimónGrima \& Roso-Moliner 2019). Por ello, los datos obtenidos en el presente estudio podrían ser extrapolables, tomándolos con precaución, a otros centros fitness de similares características en todo el territorio nacional.

Se contactó con el Manager del centro y debido a la imposibilidad de enviar una invitación a participar en el estudio vía e-mail (políticas de empresa), ésta se realizó a través de una aplicación móvil. La invitación incluyó una breve introducción al estudio, una explicación de la naturaleza anónima y voluntaria de la participación en el mismo y un link a un cuestionario online. En total, 340 usuarios potencialmente elegibles aceptaron finalmente participar en el estudio. Finalmente, 102 de éstos (52 hombres y 50 mujeres) fueron reclutados por cumplir los criterios de inclusión, los cuales incluían ser mayor de edad y asistir un mínimo de dos veces por semana al centro de fitness para practicar ejercicio físico. Se incluyó este valor tomando como referencia el American College of Sports Medicine (ACSM, 2014), el cual recomienda al menos 2-3 días de práctica de actividad física (trabajo aeróbico, fuerza, flexibilidad, equilibrio y agilidad) en adultos sanos. La edad media de la muestra fue de 29,7 $\pm 6,9$ años (rango entre los 18 y los 47 años de edad).

\section{Estatus sociodemográfico}

Se utilizó un cuestionario de elaboración propia realizado con la plataforma Google Drive, tomando como referencia el elaborado por Garcés et al. (2018). El cuestionario estuvo compuesto por preguntas relacionadas con variables como el sexo, edad, nivel educativo, estado civil, tipo de ocupación, actividades que realiza en el centro fitness, uso de dispositivos en la práctica habitual de ejercicio físico, etc. Las preguntas formuladas en éste cuestionario fueron tanto abiertas como cerradas, siendo la mayor parte de ellas del segundo tipo.

\section{Adicción al ejercicio}

Para evaluar la adicción al ejercicio se utilizó el Exercise 
Addiction Inventory (EAI), desarrollado por Szabo y Griffiths (2004), y cuya versión española ha mostrado propiedades psicométricas satisfactorias (á value $=70, \mathrm{ICC}=, 92$ ) (Sicilia, Alías-García, Ferriz \& Moreno-Murcia, 2013). Se trata de un cuestionario auto-administrado que consta de 6 cuestiones basadas en los seis componentes de la adicción: rasgo sobresaliente, modificación del estado de ánimo, tolerancia, síntomas de abstinencia, conflicto social y recaída. Las respuestas son clasificadas en una escala de 5 puntos. El EAI clasifica a los sujetos con riesgo de adicción al ejercicio alto (puntuación: 24-30), riesgo de adicción al ejercicio bajo (puntuación: 0-23) y asintomáticos (puntuación: d»12). En estudios previos realizados con sujetos practicantes de Crossfit, este instrumento ha probado ser una herramienta eficaz y válida para la evaluación de la adicción al ejercicio (Lichtenstein \& Jensen, 2016).

\section{Ansiedad rasgo}

Para evaluar el nivel de ansiedad rasgo, se usó el cuestionario validado State-Trait Anxiety Inventory (STAI), cuya primera versión fue desarrollada por Spielberger, Gorshuch $\&$ Lushene en 1968. El STAI se ha utilizado en multitud de estudios, tanto en población adulta como infantil (Spielberger \& Reheiser, 2009). Además, ha mostrado características psicométricas satisfactorias, con una alta consistencia interna y gran fiabilidad (Op. Cit). En la herramienta STAI, los sujetos deben marcar una opción para cada ítem que se quiere evaluar. La escala de respuestas de tipo Likert esta graduada de 0 (casi nunca) a 3 (casi siempre). Al final del cuestionario, el sujeto obtiene una puntuación que se encuentra en el rango de 0-60, siendo el 0 el nivel mínimo de ansiedad y el 60 el nivel máximo. La puntuación en los ítems 1,6,7,10,13,16 y 19 se invierte para realizar el sumatorio final.

\section{Análisis estadístico}

En primer lugar, se recodificaron manualmente algunas respuestas de los encuestados otorgándoles un valor numérico, para poder trabajar con ellas y poder analizarlas en los programas estadísticos adecuados. El análisis de los datos obtenidos, se llevó a cabo mediante el programa SPSS versión 23 (IBM SPSS Statistics para Windows). El programa Excel, de Microsoft Office, fue empleado principalmente para el desarrollo de gráficos representativos de los datos. Una vez analizada la normalidad de la muestra, se calcularon los descriptivos de todas las variables, expresándose mediante Media y Desviación Estándar (Media \pm DE). Posteriormente, con el objetivo de averiguar la relación entre variables cuantitativas, se utilizó el Coeficiente de Pearson. Se consideró el $p$-valor de la significación como $p<0,05$, teniendo en cuenta las tendencias a la significación en $\mathrm{p}<0,1$. Para valorar el grado de asociación, se utilizó la clasificación de Hopkins (2002), donde 0,1, trivial; 0,1-0,3, pequeña; 0,3-0,49, moderada; 0,5-0,69, grande; 0,7-0,89, muy grande; $y>0,9$ a 1 casi perfecta. Se aplicó la prueba de contraste t de Student (pruebas paramétricas) para comparar medias en las distintas variables tras comprobar que cumplían la distribución normal. Esto se realizó en las variables de EAI, ansiedad rasgo y el uso de dispositivos, y en si competían o no, tanto en hombres como en mujeres.

\section{Resultados}

La muestra del presente estudio estuvo compuesta de 102 sujetos ( 52 hombres y 50 mujeres). La edad media del total de la muestra fue de 29,29 \pm 6,90 años (rango de 18-47 años). Los hombres tuvieron una media de edad de 28,54 años, ligeramente inferior a la de las mujeres (30,28 años). Con respecto a la frecuencia de entrenamiento semanal, el $37,3 \%$ de la muestra entrenaba 3 días a la semana, y el 31,4\% dedicaban 4 días semanales a realizar ejercicio físico en el centro. No se encontraron correlaciones significativas $(\mathrm{p}>0,05)$ entre la frecuencia de entrenamiento y el uso de dispositivos fitness o entre ésta (frecuencia de entrenamiento) y la ausencia o no de competición por parte de los sujetos.

Del total de la muestra, el 68\% había utilizado algún tipo de tecnología mientras realizaba ejercicio físico en el centro fitness. El 32\% restante no utilizaba habitualmente dispositivos durante el desarrollo de las diferentes actividades. Diferenciando entre sexos, el 73\% de los hombres usaban dispositivos fitness, mientras que el porcentaje en mujeres fue inferior $(62 \%)$.

Entre los distintos tipos de dispositivos utilizados por los sujetos del estudio, el más usado fue el pulsómetro (53\%), seguido de las aplicaciones móviles (49\%), smartwatch (47\%), smartband (41\%) y el GPS (27\%). Los acelerómetros y el reloj poolmate (acuático) solo fueron usados por el 3\% de la muestra. Las zonas en las que se reportó un mayor uso de los distintos dispositivos tecnológicos fueron: musculación en sala (49\%) y zona de cardio (47\%). El $41 \%$ de la muestra usaba dispositivos tecnológicos para realizar actividades dirigidas y el 33\% para practicar running. Solamente el 3\% de los usuarios usaron dispositivos tecnológicos para practicar natación. En la figura 1 se muestran las justificaciones al uso de los distintos dispositivos fitness por parte de los sujetos y el porcentaje de éstos que se referían a ellas. Destacar que el control de la intensidad fue el motivo más expuesto por los usuarios para justificar el uso de los dispositivos tecnológicos.

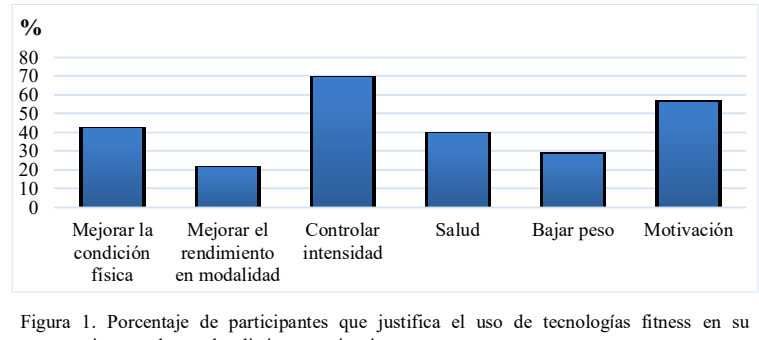
entrenamiento en base a las distintas motivaciones expuestas.

En la tabla 1 se muestran los resultados obtenidos en el cuestionario EAI y STAI en hombres y mujeres, usuarios que usan o no la tecnología fitness para entrenar, y usuarios que compiten o no compiten. Cabe destacar que el 3,9\% del total de la muestra se situaron en riesgo de adicción al ejercicio según su puntuación en el EAI (e»24 puntos). No se observaron diferencias significativas en cuanto al riesgo de adicción al ejercicio entre hombres y mujeres $(p>0,05)$. Tampoco se observaron diferencias $(p>0,05)$ entre sujetos que usaban dispositivos y/o tecnología fitness y el riesgo de 
adicción al ejercicio. En cuanto a la relación entre la ausencia o no de competición y las puntuaciones en el EAI, ésta no fue significativa $(p=0,061)$. En cuanto a las puntuaciones observadas en el STAI, no se encontraron asociaciones entre el sexo y el nivel de ansiedad rasgo. Tampoco existieron diferencias significativas $(p>0,05)$ entre usuarios que usaban o no dispositivos fitness y los niveles de ansiedad rasgo. Tampoco se observaron diferencias significativas en cuanto a la ansiedad rasgo entre sujetos que competían o no competían $(\mathrm{p}>0,05)$.

Tabla 1.

Diferencias en la puntuación del Exercise Addiction Inventory y del State Trate Anxiety Index entre hombres mujeres, entre usuarios que usan o no la tecnología fitness y entre usuarios que compiten o no compiten. \begin{tabular}{ccc}
\hline $\begin{array}{c}\text { Diferencias en función del } \\
\text { género }\end{array}$ & $\begin{array}{c}\text { Diferencias en función del uso } \\
\text { de tecnología fitness }\end{array}$ & $\begin{array}{c}\text { Diferencias en función de la } \\
\text { participación en competiciones }\end{array}$ \\
\hline
\end{tabular}

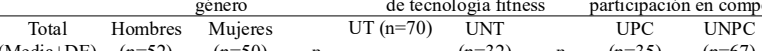

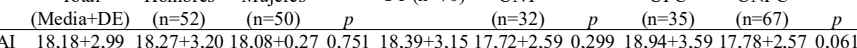

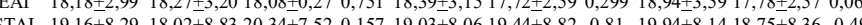

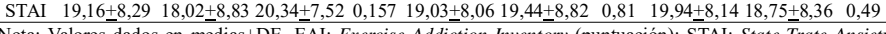
Nota: Valores dados en medias_DE. EAI: Exercise Addiction Inventory (puntuación); STAI: State Trate Ansiety Index (puntuacion); UT: Usuarios que usan tecnologia fitn

En la tabla 2 se muestran las correlaciones encontradas en el presente estudio entre diferentes variables. Cabe destacar la correlación negativa moderada entre ansiedad rasgo y edad ( $\mathrm{p}<0,01)$ (tabla 2). Asimismo, también se encontró una correlación negativa débil entre los niveles de ansiedad rasgo y los años de práctica de ejercicio físico $(\mathrm{p}<0,05)$ (tabla 2$)$. Por último, destacar la correlación positiva débil $(\mathrm{p}<0,05)$ que se dio entre la puntuación en las puntuaciones en el Exercise Addiction Inventory y los niveles de ansiedad rasgo (tabla 2). No hubo asociaciones significativas entre el riesgo de adicción al ejercicio y los años de experiencia de entrenamiento $(\mathrm{p}>0,05)$ (tabla 2$)$.

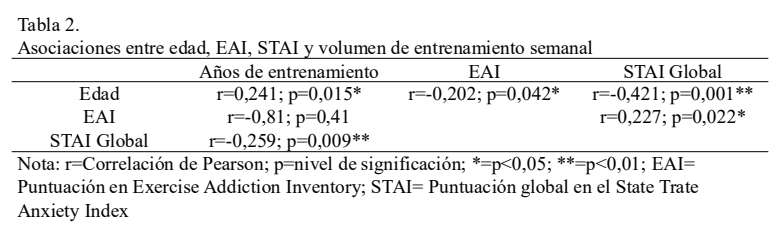

\section{Discusión}

En el presente estudio se pretendió buscar asociaciones y/o diferencias en usuarios de centros fitness entre variables relacionadas con la salud como el riesgo de adicción al ejercicio, los niveles de ansiedad rasgo, la edad, el volumen de entrenamiento, años de experiencia en entrenamiento o el uso de tecnología fitness para entrenar. Uno de los principales hallazgos fue que solamente un 3,9\% de la muestra mostró un riesgo de adicción al ejercicio (medido a través del Exercise Addiction Inventory).

\section{Variables descriptivas de la muestra}

Cabe destacar que en estudios previos como el llevado a cabo por García-Ferrando \& Llopis (2011), la edad de los participantes fue inferior a la de nuestro estudio, la cual se situó entre los rangos de 18 a 47 años. Además, en nuestro estudio se ha observado que la frecuencia y el volumen de entrenamiento es superior en hombres que en mujeres, lo que concuerda con lo observado en el estudio de Berzosa, Gómez-Angulo, Regodón \& Santamaría (2013), los cuales observaron que el tiempo dedicado al entrenamiento en hom- bres era de 6,69 horas semanales frente a las 4,49 horas semanales que practicaron las mujeres. El estudio realizado a nivel nacional por Subijana, Conde, Barriopedro \& Gallardo (2014) también concluyó que generalmente, las mujeres entrenan menos horas que los hombres. En el presente estudio también se observó que los hombres competían más que las mujeres ( $77 \%$ vs $23 \%$, respectivamente). Esto podría deberse, según del Castillo et al. (2009), a que los hombres son más autónomos en su práctica, la realizan en espacios abiertos y tienen motivaciones más competitivas, mientras que las mujeres se sienten más atraídas por actividades dirigidas, en espacios cerrados y orientadas a la salud.

\section{Uso de dispositivos y tecnología fitness}

En la muestra de nuestro estudio, aproximadamente el 68\% de la misma aseguró utilizar algún tipo de tecnología mientras realizaba ejercicio físico en el centro fitness, mientras que el $32 \%$ restante no hacía uso de éste tipo de herramientas. Estos datos son similares a los reportados en estudios previos como el realizado por Garcés et al. (2018). Además, la edad media de los sujetos de nuestro estudio que usaban tecnología para entrenar fue de 29 años. Esto concuerda con lo que afirman autores como Harnisch, Comstock \& Bruce (2014), los cuales argumentan que determinadas tecnologías tienen muchísimas más posibilidades de ser usadas por parte de los menores de 30 años que por el resto de la población. No obstante, en EEUU (país con mayor venta de dispositivos fitness) se ha demostrado que existe un público objetivo más amplio en cuanto a la edad, dependiendo del dispositivo utilizado (Hughes et al. 2015). También se ha observado en el presente estudio que los hombres utilizan más los dispositivos tecnológicos para entrenar que las mujeres. Por el contrario, estudios como el realizado por Garcés et al. (2018) mostraba resultados diferentes, reflejando un mayor uso de dispositivos por parte de las mujeres. Esto puede deberse a la limitada muestra utilizada en nuestro estudio y al hecho de ser usuarios de centros fitness y no únicamente practicantes de un determinado deporte o disciplina deportiva. No obstante, Blas (2015) concluyó en su estudio que los smartwatch eran una herramienta mucho más usada por hombres que por mujeres ( $71 \%$ vs $29 \%$, respectivamente).

Con respecto a qué dispositivo era el más usado por los sujetos de la muestra, el pulsómetro se colocó en primera posición (53\%). Ésta elección del pulsómetro como dispositivo más usado, coincide con estudios previos (Garcés et al. 2018), y puede ser debido a que ciertas tecnologías innovadoras no han sido bien aceptadas por los consumidores, puesto que no se perciben con beneficios superiores a los que reportan dispositivos precedentes (Dibb et al. 2013). Las app móviles fueron la segunda opción más utilizada (49\%), y su uso generalizado parece residir en gran medida en cuantificar la actividad física y fomentar la motivación hacia la misma (Vardus, van Beurden, Smith \& Abraham, 2016).

\section{Riesgo de adicción al ejercicio}

Los resultados obtenidos en el presente estudio muestran que la puntación media de los sujetos participantes en el Exercise Addiction Inventory (EAI) fue de 18,18 puntos (ran- 
go de 6 a 30 puntos). Esta puntuación catalogaría a esta población como sintomática, según las categorías creadas para la valoración del EAI (Griffiths et al., 2005). En el caso de nuestro estudio no existiría un riesgo de adicción al ejercicio, aunque es verdad que bajo nuestro conocimiento, existen pocos estudios relacionados con la adicción al ejercicio en poblaciones del ámbito del fitness. Uno de estos estudios fue el realizado por Liechtenstein, Emborg, Hemmingsen \& Hansen (2017), el cual arroja datos similares a los obtenidos en el presente estudio, ya que la población de la investigación de estos autores obtuvo una puntuación global media en el EAI de 17 puntos (sintomáticos). Cabe destacar que en ese estudio se tenían en cuenta otras variables distintas a las analizadas en el presente estudio, como el apoyo del círculo cercano del sujeto y su asociación con el riesgo de adicción al ejercicio. Sin embargo, a pesar de que la población estudiada en el presente estudio se clasifique como sintomática, sí que se observó que un 3,9\% del total de los encuestados sí que se encuentran en riesgo de adicción al ejercicio físico (e»24 puntos). Esto concuerda con estudios realizados en población general de personas que realizan ejercicio físico (Mónok, Berczik, Urbán, Szabo, Griffiths, Farkas \& Demetrovics 2012) y que mostraron que el 3,2\% (usando el EAI) de la muestra analizada tenía riesgo de adicción al ejercicio. En otros estudios más recientes se han observado porcentajes muy por encima de los vistos en el presente estudio, como el de Bruno et al. (2014), con un 42,5\% en riego de adicción (utilizando el EAI) en 150 usuarios de gimnasio.

Analizando la variable del sexo, en el presente estudio no hemos encontrado diferencias significativas en las puntuaciones del EAI entre hombres y mujeres. En otros estudios sí que se han visto diferencias significativas entre hombres y mujeres, por ejemplo, el estudio realizado por Cook, Hausenblas y Rossi (2012), mostró una mayor riesgo de adicción al ejercicio en hombres insatisfechos con su peso con respecto a mujeres insatisfechas con su peso. No obstante, existen investigaciones que utilizaron como muestra a triatletas en las que las mujeres presentaban una adicción al ejercicio mayor que los hombres (22 vs 18\% respectivamente) (Youngman \& Simpson, 2014). A su vez, en estudios realizados con maratonianos (también atletas de resistencia), se observó que el riesgo de adicción al ejercicio fue más alto en hombres que en mujeres (19vs 15\% respectivamente) (Szabo, de la Vega, Ruiz-Barquín \& Rivera, 2013). En el caso específico del Crossfit, actividad más enfocada al fitness, que es donde principalmente se centra nuestra población de estudio, se vio que los hombres presentaban más adicción al ejercicio que las mujeres (Lichtenstein \& Jensen, 2016).

Finalmente, concluir que en la literatura científica no existe un consenso respecto al género y su influencia en la adicción al ejercicio en la actualidad, ya que parece depender de múltiples factores como: edad, tipo de deporte y/o ejercicio realizado, volumen de entrenamiento, etc. Por lo tanto, parece ser que el riesgo de adicción al ejercicio no es similar en todas las poblaciones que realizan ejercicio físico.

Uso de dispositivos tecnológicos y riesgo de adicción al ejercicio

Cabría esperar que el uso de dispositivos y la adicción al ejercicio tuvieran relación o dependencia, sin embargo, tam- poco se observaron en el presente estudio diferencias significativas entre usuarios que usaban la tecnología fitness o no y la puntuación en el EAI. Bajo nuestro conocimiento, en la literatura científica no hemos encontrado investigaciones que relacionen estas dos variables. Se podría establecer alguna hipótesis en base a lo que se observó en el estudio realizado por Youngman \& Simpson (2014), el cual concluyó que existía una asociación positiva entre el riesgo de adicción al ejercicio y el uso de tecnología fitness en triatletas. También se observó algo similar en una muestra de ultramaratonianos (Szabo et al., 2013). Se ha visto que estos grupos de estudio, presentan un riesgo de adicción al ejercicio de entre el 17 y el $20 \%$, y esto se justificar con el hecho de que para monitorizar el trabajo cardiovascular (zona de trabajo, ritmo cardíaco, etc.) el uso de dispositivos es de gran utilidad y es empleado por gran parte de estos sujetos. No obstante, se hace necesario el desarrollo de más estudios bien diseñados en el futuro que pudieran arrojar luz sobre este tema.

\section{Ansiedad rasgo}

Los resultados globales en cuanto a la ansiedad rasgo obtenidos en la población de nuestro estudio mostraron que la media de puntuación en el STAI es de 19,16, lo que concuerda con los obtenidos en el estudio realizado por GuillénRiquelme \& Buela (2011), en el que se realizó una actualización del State-Trait Anxiety Inventory (STAI), y el promedio de puntuación fue de 21,15 en ansiedad rasgo para población española.

En el presente estudió se pretendió establecer algún tipo de relación entre el uso de dispositivos fitness y ansiedad rasgo. Sin embargo no se han encontrado diferencias significativas entre su utilización y mayores puntuaciones en el test STAI.

No se observaron diferencias significativas entre el nivel ansiedad rasgo y el hecho de competir o no en eventos deportivos. Sin embargo, existe cierta tendencia, ya que las puntuaciones medias en el STAI son ligeramente superiores en el grupo de competidores. En un estudio realizado sobre competidores tanto de deportes individuales como colectivos se obtuvieron resultados similares con puntuaciones promedio de 19,5 en ansiedad rasgo (González-Boto, Márquez, Salguero \& Tuero, 2009). Al igual que en otro estudio anterior realizado por Guillén-García \& Sánchez-Luján y Márquez Rosa (2003), en el que también se encontraron puntuaciones similares $(18,4)$ en jugadoras de equipos de baloncesto femenino.

En el presente estudio también se halló una correlación negativa moderada entre los niveles de ansiedad-rasgo y la edad $(\mathrm{r}=-0,421, \mathrm{p}=0,000)$. Esta correlación muestra que, al aumentar la edad, se produce una disminución de las puntuaciones de ansiedad rasgo. Según estos resultados, parece ser que quizás los sujetos más adultos presenten menos ansiedad que los sujetos jóvenes. Algunos estudios han concluido que la ansiedad está muy vinculada a la imagen corporal ya el autoconcepto (Raich, 2004), y en la adolescencia es cuando más se acusa este hecho, como se vio en un estudio con nadadoras adolescentes (Camacho-Miñano \& Aragón, 2014).

Con respecto a la relación entre los niveles de ansiedad- 
rasgo y el riesgo de adicción al ejercicio, se encontró en el presente estudio una pequeña correlación positiva, según la clasificación de Hopkins (2002), $(r=0,227, p=0,022)$. En concordancia con esto, Griffiths (2005) afirma que en los síntomas de la adicción al ejercicio aparece la ansiedad cuando el ejercicio físico ocupa predominantemente los pensamientos del sujeto o cuando finalmente no se logra realizar actividad física, con lo cual, a mayor riesgo de adicción al ejercicio, es posible que aparezca una mayor ansiedad. En consonancia con el presente estudio, la investigación llevada a cabo por Spano (2001) asoció el riesgo de adicción al ejercicio con la ansiedad. A su vez, en otro estudio más reciente realizado por Li, Nie \& Ren (2015) se concluyó que el grupo con dependencia al ejercicio presentaba mayores valores de ansiedad estado, pero no en la dimensión de ansiedad rasgo, que es la analizada en nuestro estudio.

\section{Edad y riesgo de adicción al ejercicio}

Hallamos una pequeña correlación negativa entre la edad y la adicción al ejercicio físico evaluada a través del EAI ( $\mathrm{r}=$ $0,202, p=0,042$ ). Conforme aumenta la edad del sujeto, su adicción al ejercicio disminuiría, y viceversa. Aunque esta relación es muy pequeña, queremos resaltarla puesto que no son muchos los estudios que han analizado la asociación entre edad y adicción al ejercicio. No obstante, investigaciones como la llevada a cabo por Furst \& Germone (1993) y algunas más recientes como la realizada por Mayolas et al. (2018), muestran resultados no concuerdan con lo observado en el presente estudio. En contraposición a esto, estudios como el de Allegre, Therme \& Griffiths (2007), realizado en 95 ultramaratonianos y de Lindwall \& Palmeira (2009), con una muestra de deportistas de élite, practicantes de ejercicio en gimnasios y estudiantes de ciencias del deporte, mostraron que los individuos que no tenían síntomas de dependencia al ejercicio eran de mayor edad. Cabe destacar que el estudio realizado por Mayolas et al. (2018) se centró en una muestra de ciclistas (no usuarios de centros fitness), más amplia que la usada en el presente estudio. Además, la correlación encontrada en nuestro estudio es leve, por lo tanto, estos resultados deberían de analizarse con mucha precaución. Creemos en todo caso de interés seguir estudiando la relación entre las variables mencionadas.

\section{Conclusión}

En relación de los usuarios de los centros de fitness valorados, casi un $4 \%$ se sitúa en riesgo de adicción al ejercicio $(3,9 \%)$. Por otro lado, este riesgo de adicción al ejercicio parece estar relacionado con mayores niveles de ansiedadrasgo y con una menor edad. Sin embargo, no parece estar asociado con el sexo o con el mayor o menor uso de tecnología fitness para el entrenamiento. Con respecto a los niveles de ansiedad-rasgo, parece que las personas de mayor edad tienen niveles más bajos de ansiedad-rasgo. No obstante, estos niveles de ansiedad-rasgo no se asocian con el uso de tecnología fitness, el sexo o la participación en eventos competitivos o no.

\section{Conflicto de interés}

Los autores declaran la inexistencia de conflictos de in- tereses respecto de este análisis.

\section{Referencias}

ACSM's guidelines for exercise testing and prescription. Ninth edition (2014). American College of Sports Medicine. Recuperado el 6 de Abril de 2020 a partir de http://antoinedl.com/ fichiers/public/ACSM-guidelines-2014.pdf

Aladro-Gonzalvo, A. R., \& Ibarzábal, F. A. (2019). Tecnología digital para la salud y la actividad física. Ediciones Pirámide.

Allegre, B., Therme, P., \& Griffiths, M. (2007). Individual factors and the context of physical activity in exercise dependence: A prospective study of 'ultra-marathoners'. International Journal of Mental Health and Addiction, 5(3), 233-243.

American Psychiatric Association. (2013). Diagnostic and statistical manual of mental disorders (DSM-5®). American Psychiatric Pub.

Araújo, S. R. C. D., Mello, M. T. D., \& Leite, J. R. (2007). Transtornos de ansiedade e exercício físico. Brazilian Journal of Psychiatry, 29(2), 164-171.

Bardus, M., van Beurden, S. B., Smith, J. R., \& Abraham, C. (2016). A review and content analysis of engagement, functionality, aesthetics, information quality, and change techniques in the most popular commercial apps for weight management. International Journal of Behavioral Nutrition and Physical Activity, 13(1), 35.

Berzosa, J., Gómez-Angulo, J., Regodón, C., \& Santamaría, L. (2013). ¿Te cuidas? Hábitos de vida de las mujeres de la Comunidad de Madrid. Madrid: Comunidad de Madrid, Consejería de Asuntos Sociales.

Blas, C. (2015). Estudio de accesorios wearable, sus capacidades y la comunicación con un smartphone (Doctoral dissertation, Universitat Politècnica de Catalunya. Escola d'Enginyeria de Telecomunicació i Aeroespacial de Castelldefels, 2015 (Grau en Enginyeria Telemàtica).

Bruno, A., Quattrone, D., Scimeca, G., Cicciarelli, C., Romeo, V. M., Pandolfo, G. \& Muscatello, M. R. A. (2014). Unraveling exercise addiction: the role of narcissism and self-esteem. Journal of Addiction, 2014. doi:10.1155/2014/987841

Camacho-Miñano, M. J., \& Aragón, H. (2014). Social physical anxiety and school physical education: teenage girls in swimming lessons. Apunts. Educació Fisica i Esports, (116), 87-94.

Cook, B., Hausenblas, H., \& Rossi, J. (2013). The moderating effect of gender on ideal-weight goals and exercise dependence symptoms. Journal of Behavioral Addictions, 2(1), 50-55.

de Subijana, C. L., Conde, E., Barriopedro, M. I., \& Gallardo, L. (2014). Eficacia en la aplicación de las medidas de formación para los deportistas de alto nivel en el territorio español. Revista de Psicología del Deporte, 23(1), 41-48.

del Castillo, J. M., Rivera, M. D. G., Navarro, J. E. J. B., Sanz, J. L. G., Rodríguez, M. M., Izquierdo, A. C., \& Pinés, D. D. H. (2009). Los hábitos de actividad física de las mujeres mayores en España. RICYDE. Revista Internacional de Ciencias del Deporte, (14), 81-93.

Dibb, S., Carrigan, M., Zainuddin, N., Russell-Bennett, R., \& Previte, J. (2013). The value of health and wellbeing: an empirical model of value creation in social marketing. European Journal of Marketing.

Estrada-Marcén, N. C., Sanz-Gonzalo, G., Simón-Grima, J., Casterad-Seral, J., \& Roso-Moliner, A. (2019a). Los centros de fitness de la ciudad de Zaragoza. Apunts Educación Física y Deportes, 35(135), 118-136.

Estrada-Marcén, N. E., Gonzalo, G. S., Seral, J. C., Simón-Grima, J., \& Moliner, A. R. (2019b). Perfil profesional de los trabajadores del sector del fitness en la ciudad de Zaragoza. Retos: nuevas tendencias en educación física, deporte y recreación, (35), 185-190. 
Ferguson, B. (2014). ACSM's guidelines for exercise testing and prescription 9th Ed. 2014. The Journal of the Canadian Chiropractic Association, 58(3), 328.

Furst, D. M., \& Germone, K. (1993). Negative addiction in male and female runners and exercisers. Perceptual and motor skills, 77(1), 192-194.

Garcés, T. E., Ortega, F. Z., Cuberos, R. C., Sánchez, M. C., Martínez, A. M., \& Cortés, A. J. P. (2018). Actividad física y autoconcepto: dos factores de estudio en adolescentes de zona rural. Revista iberoamericana de psicología del ejercicio y el deporte, 13(2), 203-210.

García-Ferrando, M., \& Llopis, R. (2011). Ideal democrático y bienestar personal. Encuesta sobre los hábitos deportivos en España 2010. Madrid: Consejo Superior de Deportes, Centro de Investigaciones Sociológicas.

González-Boto, R., Salguero, A., Tuero, C., \& Márquez, S. (2009). Validez concurrente de la versión española del Cuestionario de Recuperación-Estrés para Deportistas (RESTQ-Sport). Revista de psicología del deporte, 18(1), 53-72.

Griffiths, M. (1996). Behavioural addiction: an issue for everybody? Journal of workplace Learning, 8(3):19-25.

Guillén García, F., Sánchez Luján, R., \& Márquez Rosa, S. (2003). La ansiedad en jugadores de la Liga Española de Baloncesto. Archivos de medicina del deporte, 5, 213-220

Guillén-Riquelme, A., \& Buela-Casal, G. (2011). Actualización psicométrica y funcionamiento diferencial de los ítems en el State Trait Anxiety Inventory (STAI). Psicothema, 23(3), 510515.

Harnisch, D. L., Comstock, S. L., \& Bruce, B. C. (2014). Collaborative inquiry with technology in secondary science classrooms: Professional learning community development at work. E-Learning and Digital Media, 11(5), 495-505.

Hollander, E. (1993). Obsessive-compulsive spectrum disorders: an overview. Psychiatric Annals, 23(7), 355-358

Hopkins WG. A scale of magnitudes for effect statistics. Anew view of statistics. 2002;502:411

Hortas-Rico, M., Onrubia, J. \& Pacifico, D. (2014). Estimating the Personal Income Distribution in Spanish Municipalities Using Tax Micro-Data. International Center for Public Policy, Andrew Young School of Policy Studies, Georgia State University. Recuperado el 6 de Abril de 2020 a partir de https:// scholarworks.gsu.edu/icepp/13

Hughes, C. E., Nagendran, A., Dieker, L. A., Hynes, M. C., \& Welch, G. F. (2015). Applications of avatar mediated interaction to teaching, training, job skills and wellness. In Virtual Realities (pp. 133-146). Springer, Cham.

Kokkinos, P., \& Myers, J. (2010). Exercise and physical activity: clinical outcomes and applications. Circulation, 122(16), 1637 1648 .

Lader M. (2015) Generalized Anxiety Disorder. In: Stolerman I.P., Price L.H. (eds) Encyclopedia of Psychopharmacology. Springer, Berlin, Heidelberg

Lejoyeux, M., Guillot, C., Chalvin, F., Petit, A., \& Lequen, V. (2012). Exercise dependence among customers from a Parisian sport shop. Journal of Behavioral Addictions, 1(1), 28-34.

Li, M., Nie, J., \& Ren, Y. (2015). Effects of exercise dependence on psychological health of Chinese college students. Psychiatria Danubina, 27(4), 0-419.

Lichtenstein, M. B., \& Jensen, T. T. (2016). Exercise addiction in CrossFit: Prevalence and psychometric properties of the Exercise Addiction Inventory. Addictive behaviors reports, 3, 33-37.

Lichtenstein, M. B., Emborg, B., Hemmingsen, S. D., \& Hansen, N. B. (2017). Is exercise addiction in fitness centers a socially accepted behavior?. Addictive behaviors reports, 6, 102-105.

Lindwall, M., \& Palmeira, A. (2009). Factorial validity and invariance testing of the Exercise Dependence Scale-Revised in
Swedish and Portuguese exercisers. Measurement in Physical Education and Exercise Science, 13(3), 166-179.

Lopez-Ruiz, N., Lopez-Torres, J., Rodríguez, M. Á. C., de VargasSansalvador, I. P., \& Martinez-Olmos, A. (2015). Wearable system for monitoring of oxygen concentration in breath based on optical sensor. IEEE Sensors Journal, 15(7), 4039-4045.

Martinsen, E. W. (2008). Physical activity in the prevention and treatment of anxiety and depression. Nordic journal of psychiatry, 62(sup47), 25-29.

Mayolas-Pi, C., Simon-Grima, J., Penarrubia-Lozano, C., MunguiaIzquierdo, D., Moliner-Urdiales, D., \& Legaz-Arrese,A. (2016). Exercise addiction risk and health in male and female amateur endurance cyclists. Journal of Behavioral Addictions, 6(1), 7483

Mónok, K., Berczik, K., Urbán, R., Szabo, A., Griffiths, M. D., Farkas, J. y Demetrovics, Z. (2012). Psychometric properties and concurrent validity of two exercise addiction measures: A population wide study. Psychology of Sport and Exercise, 13, 739-746. doi: 10.1016/j.psychsport.2012.06.003

Raich, R. M. (2004). Una perspectiva desde la psicología de la salud de la imagen corporal. Avances en psicología Latinoamericana, 22(1), 15-27.

Riebe, D., Ehrman, J. K., Liguori, G., Magal, M., \& American College of Sports Medicine (Eds.). (2018). ACSM's guidelines for exercise testing and prescription. Wolters Kluwer.

Sicilia, A., Alías-Garcia, A., Ferriz, R., \& Moreno-Murcia, J. A. (2013). Adaptación y validación al español del Inventario de Adicción al Ejercicio (EAI). Psicothema, 25(3), 377-384.

Simon-Grima, J., Estrada-Marcen, N., \& Montero-Marín, J. (2019). Exercise addiction measure through the Exercise Addiction Inventory (EAI) and health in habitual exercisers. A systematic review and meta-analysis. Adicciones, 31(3), 233-249.

Smith, A. (2015). U.S. Smartphone Use in 2015 |Pew Research Center Pew Internet \& American Life Project, 59. Recuperado 6 de abril de 2020, a partir de https://www.pewinternet.org/ 2015/04/01/us-smartphone-use-in-2015/

Spano, L. (2001). The relationship between exercise and anxiety, obsessive-compulsiveness, and narcissism. Personality and Individual Differences, 30(1), 87-93.

Spielberger, C. D., \& Reheiser, E. C. (2009). Assessment of emotions: Anxiety, anger, depression, and curiosity. Applied Psychology: Health and Well Being, 1(3), 271-302.

Subijana, C., Conde, E., Barriopedro, M.I. \& Gallardo, L. (2014). Eficacia en la aplicación de las medidas de formación para los deportistas de alto nivel en el territorio español. Revista de Psicología del deporte 23(1), 41-48.

Egorov, A. Y., \& Szabo, A. (2013). The exercise paradox: An interactional model for a clearer conceptualization of exercise addiction. Journal of Behavioral Addictions, 2(4), 199-208. doi:10.1556/JBA.2.2013.4.2

Szabo, A., \& Griffiths, M. D. (2004). The exercise addiction inventory: A new brief screening tool. Addiction research and theory, 12(5), 489-499.

Szabo, A., De La Vega, R., Ruiz-Barquín, R., \& Rivera, O. (2013). Exercise addiction in Spanish athletes: Investigation of the roles of gender, social context and level of involvement. Journal of behavioral addictions, 2(4), 249-252.

Szabo, A., Griffiths, M. D., De la Vega-Marcos, R, Mervó, B., \& Demetrovics, Z. (2015). Focus: addiction: methodological and conceptual limitations in exercise addiction research. The Yale journal of biology and medicine, 88(3), 303.

Warner, R., \& Griffiths, M. D. (2006). A qualitative thematic analysis of exercise addiction: An exploratory study. International Journal of Mental Health and Addiction, 4(1), 13-26.

Youngman, J., \& Simpson, D. (2014). Risk for exercise addiction: A comparison of triathletes training for sprint-, Olympic-, halfironman-, and ironman-distance triathlons. Journal of Clinical Sport Psychology, 8(1), 19-37. 